\title{
The Growing Need for Both High Tech and High Touch in Today's Healthcare Approaches
}

Barb Himes, IBCLC

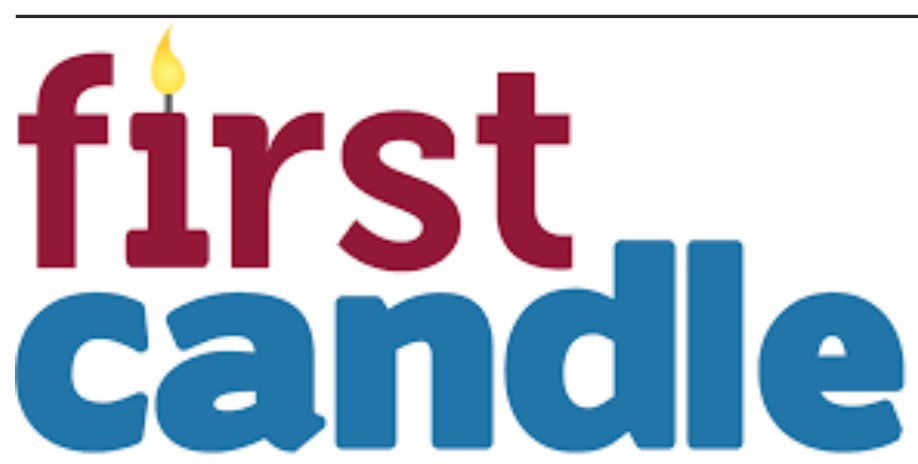

Saving babies. Supporting families.

First Candle's efforts to support families during their most difficult times and provide new answers to help other families avoid the tragedy of the loss of their baby are without parallel.

\section{"The Covid-19 pandemic has triggered} a decline in pediatric vaccination rates across the country, as parents avoid taking their children into clinics or hospitals for fear of coming into contact with the coronavirus."

One of the observations from the virtual 2020 Michigan Maternal Health Summit held in September, hosted by The Michigan Department of Health \& Human Services, the Michigan Alliance for Innovation of Maternal Health (MI-AIM) \& Maternal Infant Health (1), was that while we are now physically distanced, we are socially connected.

There is something hopeful-sounding in that, but in the maternal and infant health care arena, it is also a call to address change and look at what being socially connected really means. We do not know how long the Covid-19 pandemic and the efforts to mitigate it will last, but it is fair to say it is likely to last longer than originally thought and that even when it begins to lift, things may still not go back to where they were before.

First Candle is anecdotally learning about shifts in behavior and care approaches through our Straight Talk for Infant Safe Sleep program, which works with health care professionals around the American Academy of Pediatrics' (AAP) safe sleep practices. These include the physical sleep environment for infants, the importance of maintaining maternal wellness visits, the benefits of breastfeeding, and the threat to maternal and infant health from health inequities and implicit bias.

\section{What Is Emerging and Actions to Consider}

- More mothers appear to be receptive to infant care information from providers because traditional influences from family members have, in some cases, been interrupted, as extended family members sometimes cannot visit at will due to pandemic distancing. This gives providers an opportunity but also a responsibility to foster a relationship through patient-centered approaches and implicit bias awareness.

- $\quad$ There are indications that breastfeeding is on the rise with mothers and newborns in hospitals before they are sent home. This is believed to stem from mothers having more quiet time due to curtailed visits. This may lead hospitals to reexamine their visitation policies long-term, to build in this "mom time."

- $\quad$ Compliance with wellness visits may be affected due to concerns about onsite clinic appointments and hitches in adapting to telemedicine practices on the part of both patients and physicians. Telemedicine, or telehealth, is here to stay and offers both benefits and challenges around accessibility, scheduling and providers learning how to develop a personal, "high touch" (as opposed to a "high tech") approach.

- The pandemic underscores the importance of paternal involvement throughout the birth process while at the same time, making it harder in some cases for this to happen. Some fathers may have jobs that cannot be done from home, may not be able to take time off, and may be dealing with their own concerns around Covid-19 exposure in the workplace.

As we noted in July's issue (2), paternal involvement has been

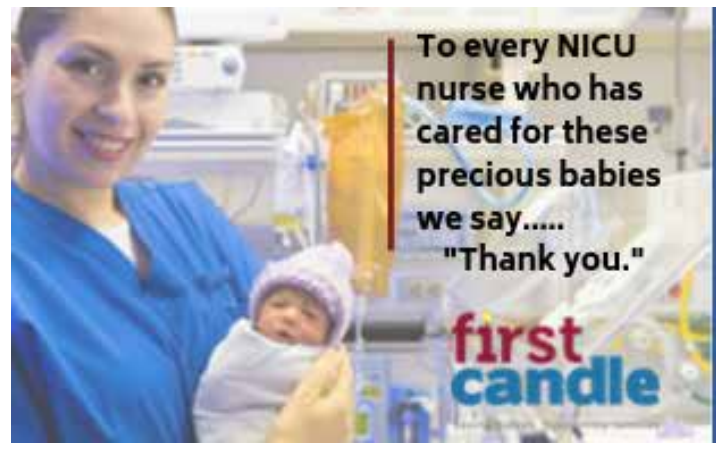

Did you know that premature and low birth weight babies have a $4 x$ greater risk for SIDS?

At First Candle we're educating parents, grandparents and caregivers about safer sleep to make sure all babies reach their first birthday. Learn more at firstcandle.org 
shown to have a positive effect on maternal health behaviors and reduced risk of preterm birth, low birth weight, and infant mortality up to one year after birth, so efforts to work around limitations and incorporate them matter.

\section{Patient-Centered Care}

Another theme from the Michigan Summit (3) was the critical importance of a patient-centered approach. There can be an unwitting tendency on the part of providers to enter the patient-provider relationship as the expert, losing an opportunity to form a productive bond of mutual understanding with the patient and her partner.

In a regional qualitative survey presented at the Summit, respondents reported feeling a lack of relationship with healthcare providers and implicit bias. Perceptions included being rushed or dismissed during appointments; being judged by socioeconomic status or the number of children; and medical conditions not being addressed, which subsequently worsened.

Patients who appear to be non-compliant trigger the need to look further and determine if this is due to obstacles that can be addressed. Has she or her partner lost their job due to the pandemic? Are they making ends meet? Do they have transportation or online access? Can they get time off work? Are there problems or dangers in the relationship? Is she dealing with the consequences of adverse life experiences? There can be a veil to be lifted on non-compliance.

First Candle is also seeking to lift the veil as it advances its initiative to provide community feedback to the AAP as it develops the latest update to its Safe Sleep Guidelines, due to be released in 2021. A key part of this is convening community task forces in three states, including parents, grandparents, in-home health care providers, social service agencies, and doulas. They will share feedback on the Guidelines, the challenges and objections that exist in adopting them, and the role systemic racism plays in maternal and infant health and adoption of infant safe sleep practices. The AAP revisions will then be shared with the task forces, who will explore the best ways of effectively disseminating this information to their communities. These sessions were to be in person and will now have to be virtual, and extra care will need to be taken to create a personal connection throughout.

We realize that the Covid-19 crisis adds to the challenges the healthcare community is dealing with as providers work to improve health outcomes for mothers and babies. We do not yet know the extent of the economic impact as the pandemic continues, nor of the further threats to our population's health. And when a crisis like this occurs, it strains a system that is already trying to

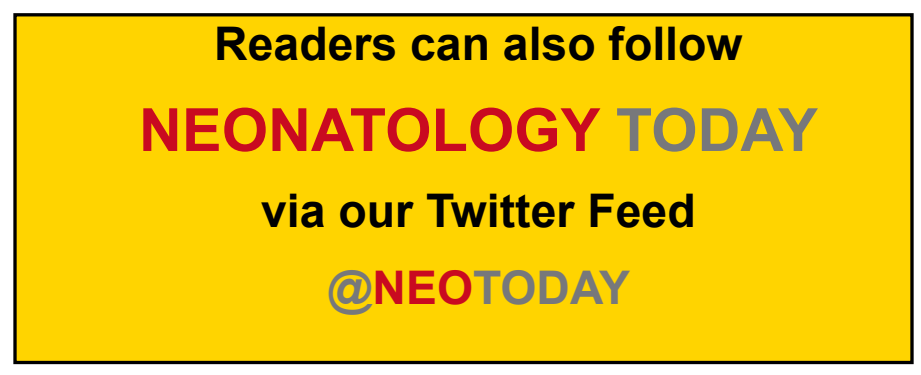

stop mortality and morbidity resulting from health inequities due to racial and socioeconomic bias. Technology will play a bigger role, but with its great benefits will also come new challenges to work through.

However, as a presenter at the Michigan Summit summed it up: "We do the best we can until we know better, and then we do better."

\section{References}

1. 2020 Maternal Infant Health Summit, September 22 - 23, 2020. https://whova.com/portal/webapp/healt 202009/

2. Examining the Benefits of Paternal Involvement and Breaking the Myths Surrounding Fathers. Neonatology Today July 2020. https://www.neonatologytoday.net/newsletters/nt-jul20. pdf

3. Through the Patient's Eyes: Washtenaw County Prenatal Survey. https://tinyurl.com/y8382ve6

Disclosure: The author is the Director of Education and Bereavement Services of First Candle, Inc., a Connecticut not for profit 501c3 corporation.

NT

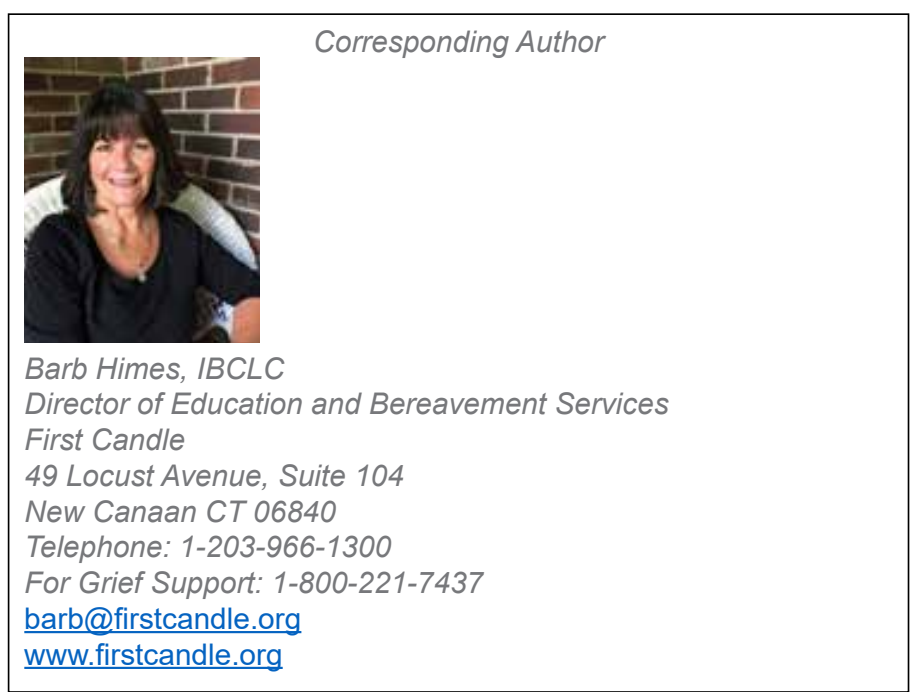

New subscribers are always welcome! NEONATOLOGY TQDAY To sign up for free monthly subscription, just click on this box to go directly to our subscription page

\section{Time is precious, just like your patients.}

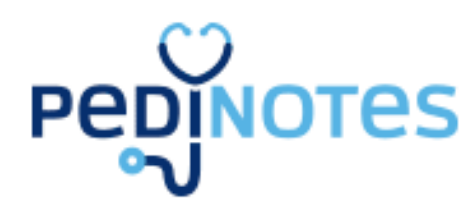

